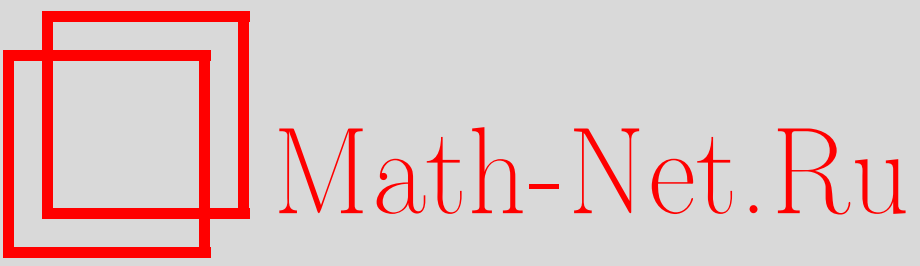

А. А. Арсеньев, О связи между полюсом матрицы рассеяния и коэффициентами прохождения и отражения при рассеянии в квантовом волноводе, ТМФ, 2004, том 140, номер 2, 303-309

DOI: https://doi.org/10.4213/tmf88

Использование Общероссийского математического портала Math-Net.Ru подразумевает, что вы прочитали и согласны с пользовательским соглашением

http://www.mathnet.ru/rus/agreement

Параметры загрузки:

IP : 54.237 .206 .68

26 апреля 2023 г., 14:13:51 
ТЕОРЕТИЧЕСКАЯ

И МАТЕМАТИЧЕСКАЯ

ФИЗИКА

Том 140, № 2

август, 2004

(C) 2004 г.

А. А. Арсеньев*

\title{
О СВЯЗИ МЕЖДУ ПОЛЮСОМ МАТРИЦЫ РАССЕЯНИЯ И КОЭФФИЦИЕНТАМИ ПРОХОЖДЕНИЯ И ОТРАЖЕНИЯ ПРИ РАССЕЯНИИ В КВАНТОВОМ ВОЛНОВОДЕ
}

\author{
На примере рассмотрено влияние полюса с малой мнимой частью на коэффициенты \\ отражения и прохождения при рассеянии в квантовом волноводе.
}

Ключевые слова: матрица рассеяния, резонансы, квантовый бильярд.

\section{1. ВВЕДЕНИЕ}

Рассматриваемая нами задача рассеяния связана с описанием баллистической проводимости в полупроводниковых гетороструктурах.

Предназначенные для детального сравнения с экспериментом расчеты свойств полупроводниковых гетороструктур основаны на кинетических моделях и очень сложны. В том случае, если длина свободного пробега носителя заряда много больше характерных размеров системы, возможен упрошенный подход, при котором проводимость трактуется как перенос заряда в поле заданного потенциала и вычисляется по формуле Ландауэра через матрицу рассеяния. Эта модель называется моделью баллистической проводимости (см. обзор [1]).

Практика показывает, что в пределах своей применимости модель баллистической проводимости в обших чертах правильно описывает основные свойства проводимости как функции напряжения. Модель баллистической проводимости проста и легко позволяет делать выводы качественного характера, что сделало ее и связанные с ней понятия квантового волновода, квантовых проволок, квантовых точек, бильярдов и т.д. популярными темами исследований, особенно в работах по физике квантовых компьютеров. Эта же модель стимулировала постановку новых задач в теории рассеяния. Основным методом исследования модели баллистической проводимости является численньй эксперимент. Если численный эксперимент показывает, что проводимость как функция напряжения имеет четко выраженный резонанс, то возникает обращенный к теории

* Московский государственный университет, Москва, Россия.

E-mail: arsenev@afrodita.phys.msu.su, arsenev@math356.phys.msu.su 
рассеяния вопрос: чем этот резонанс обусловлен? В теории рассеяния резонанс обычно связывается с полюсом аналитического продолжения матрицы рассеяния, рассматриваемым как возмущение погруженного в непрерывный спектр собственного значения гамильтониана. На примере мы покажем, что в квантовом волноводе влияние полюса матрицы рассеяния на коэффициенты прохождения и отражения может противоречить наивной физической интуиции. Это кажется нам интересным, поскольку модель квантового волновода часто используется в эвристических рассуждениях. Основной результат работы сформулирован в теореме. В связи с этим интересно сопоставить наш результат с рассуждениями, приведенными в работе [2] (абзац после формулы (2.1)). Мы будем опираться на предложенную в работе [3] модель образования резонансов. Данную работу можно рассматривать как иллюстрацию доказанной в работе [3] теоремы 2.

\section{2. ОПИСАНИЕ МОДЕЛИ}

Пусть положительные числа $h, h_{1}, d, d_{1}, l, l_{1}$ удовлетворяют условиям $0 \leqslant h \leqslant h_{1}$, $0<d<d_{1}, 0<l<l_{1}$, и пусть $0 \leqslant \epsilon<l_{1}-l$. Будем обозначать символом $\{A, B, C, \ldots\}$ ломаную, состоящую из отрезков $A B, B C, \ldots$ В пространстве $\mathbb{R}^{2}$ рассмотрим открытую область $D(\epsilon, h)$, симметричную относительно оси $x=0$, граница которой в правой полуплоскости состоит из трех ломаных:

$$
\begin{aligned}
& \left\{(0, h),(l, h),\left(l, h_{1}\right),\left(\infty, h_{1}\right)\right\}, \\
& \left\{(0,0),\left(l_{1}-\epsilon, 0\right),\left(l_{1}-\epsilon,-d\right),(0,-d)\right\}, \\
& \left\{\left(0,-d_{1}\right),\left(l_{1},-d_{1}\right),\left(l_{1}, 0\right),(\infty, 0)\right\} .
\end{aligned}
$$

Область $D(\epsilon, h)$ есть волновод (полоса $\left\{x, y ;-\infty<x<\infty, 0<y<h_{1}\right\}$ ), в который вставлена перегородка-прямоугольник с границей

$$
\left\{\left(-l, h_{1}\right),\left(l, h_{1}\right),(l, h),(-l, h),\left(-l, h_{1}\right)\right\}
$$

и добавлен резонатор - прямоугольник с границей

$$
\left\{\left(-l_{1},-d\right),\left(l_{1},-d\right),\left(l_{1},-d_{1}\right),\left(-l_{1},-d_{1}\right),\left(-l_{1},-d\right)\right\},
$$

соединенный с волноводом каналами связи (leads): прямоугольником с границей

$$
\left\{\left(l_{1}-\epsilon, 0\right),\left(l_{1}, 0\right),\left(l_{1},-d\right),\left(l_{1}-\epsilon,-d\right),\left(l_{1}-\epsilon, 0\right)\right\}
$$

и симметричным ему прямоугольником.

Пусть $L(\epsilon, h)$ - отвечаюшее граничным условиям Дирих ле на $\partial D(\epsilon, h)$ самосопряженное в $L^{2}(D(\epsilon, h))$ расширение оператора Лапласа $(-\Delta)$. Мы рассмотрим два случая:

а) перегородки нет, т.е. $h=h_{1}$,

б) перегородка полностью перекрывает волновод, т.е. $h=0$. 
В каждом из этих случаев спектр оператора $L(0, h)$ состоит из непрерывного спектра, заполняющего полуось $\left[\left(\pi / h_{1}\right)^{2}, \infty\right)$, и собственных значений $\left\{\omega_{j}\right\}$ - упорядоченного в порядке возрастания множества чисел вида

$$
\left\{\omega_{j}\right\}=\left\{\left(\frac{\pi}{d_{1}-d}\right)^{2} p^{2}+\left(\frac{\pi}{2 l_{1}}\right)^{2} q^{2}, \quad p, q \in \mathbb{Z}\right\} .
$$

Пусть $\omega_{\text {res }} \in\left\{\omega_{j}\right\}-$ простое собственное значение и

$$
\left(\frac{\pi}{h_{1}}\right)^{2}<\omega_{\text {res }}<4\left(\frac{\pi}{h_{1}}\right)^{2}
$$

В силу условия (1) число $\omega_{\text {res }}$ есть погруженное в непрерьвный спектр простое собственное значение оператора $L(0, h)$. Мы изучим поведение матришы рассеяния для пары операторов $L(\epsilon, h), L\left(0, h_{1}\right)$ в окрестности спектрального параметра $\lambda=\omega_{\text {res }}$ при малых $\epsilon$ и $h=h_{1}, h=0$. Наше основное предположение состоит в том, что при всех $\epsilon \in\left(0, \epsilon_{0}\right)$, $h=0, h=h_{1}$ у оператора $L(\epsilon, h)$ в малой окрестности точки $\omega_{\text {res }}$ нет собственных значений, т.е. в том, что при присоединении резонатора к волноводу отвечающее собственному значению $\omega_{\text {res }}$ собственное колебание резонатора преврашается в резонанс, а не в захваченную моду. При этом условии мы докажем, что при $\epsilon>0$ в окрестности точки $\omega_{\text {res }}$ сушествуют такие значения спектрального параметра $\lambda$, для которых у оператора $L\left(\epsilon, h_{1}\right)$ коэффициент прохождения почти равен нулю, а у оператора $L(\epsilon, 0)$ при $\lambda$ из той же окрестности точки $\omega_{\text {res }}$ коэффициент прохождения почти равен единице.

Это наш основной результат, и он сформулирован в теореме.

Напомним определение коэффициентов отражения и прохождения в нашей задаче. Пусть

$$
\nu_{m}=\left(\frac{\pi m}{h_{1}}\right)^{2}, \quad m=1,2, \ldots
$$

Положим

$$
e( \pm, m, \lambda, x, y)=\exp \left( \pm i x \sqrt{\lambda-\nu_{m}}\right)\left(\left(\frac{2}{h_{1}}\right)^{1 / 2} \sin \frac{\pi m y}{h_{1}}\right) \theta\left(\lambda-\nu_{m}\right)
$$

где

$$
\theta(\lambda)= \begin{cases}1, & \lambda>0 \\ 0, & \lambda \leqslant 0\end{cases}
$$

Решением стационарной задачи рассеяния для оператора $L$ будем называть функцию $u( \pm, m, \lambda, x, y)$, которая удовлетворяет следующему уравнению и граничным условиям:

$$
L_{x, y} u(\cdot, x, y)=\lambda u(\cdot, x, y), \quad(x, y) \in D(\epsilon, h), \quad u(\cdot, x, y)=0, \quad(x, y) \in \partial D(\epsilon, h)
$$

и представима в виде

$$
u( \pm, m, \lambda, x, y)=e( \pm, m, \lambda, x, y)+w( \pm, m, \lambda, x, y) .
$$

5 Теоретическая и математическая физика, т. 140, № 2, 2004 г. 
Здесь функция $w$ удовлетворяет асимптотическим соотношениям

$$
\begin{aligned}
w(+, m, \lambda, x, y) & =\sum_{p}\left[t(+, p, m, \lambda)-\delta_{p m}\right] e(+, p, \lambda, x, y)+O(\ldots), \quad x>0, \\
w(+, m, \lambda, x, y) & =\sum_{p} r(+, p, m, \lambda) e(-, p, \lambda, x, y)+O(\ldots), \quad x<0, \\
w(-, m, \lambda, x, y) & =\sum_{p} r(-, p, m, \lambda) e(+, p, \lambda, x, y)+O(\ldots), \quad x>0, \\
w(-, m, \lambda, x, y) & =\sum_{p}\left[t(-, p, m, \lambda)-\delta_{p m}\right] e(-, p, \lambda, x, y)+O(\ldots), \quad x<0, \\
O(\ldots) & =O\left(e^{-\delta|x|}\right), \quad|x| \rightarrow \infty .
\end{aligned}
$$

Суммирование в этих формулах ведется по значениям индекса $p$, удовлетворяющим условию $\nu_{p}<\lambda$. Коэффициенты $t( \pm, p, m, \lambda), r( \pm, p, m, \lambda)$ называются коэффициентами прохождения и отражения. Их физический смысл обсуждается в работе [1].

Пусть $\epsilon_{n} \rightarrow+0, n \rightarrow \infty$. Будем отмечать индексом $n$ величины, которые по описанным выше построениям соответствуют области $D\left(\epsilon_{n}, h\right)$. В дальнейшем мы будем считать, что спектральный параметр $\lambda$ удовлетворяет условию

$$
\left(\frac{\pi}{h_{1}}\right)^{2}<\lambda<4\left(\frac{\pi}{h_{1}}\right)^{2} .
$$

В силу условия (6) в правых частях уравнений (5) будет только по одному слагаемому, и мы положим

$$
\begin{aligned}
& t_{n}( \pm, \lambda, h)=t( \pm, 1,1, \lambda) \\
& r_{n}( \pm, \lambda, h)=r( \pm, 1,1, \lambda)
\end{aligned}
$$

если правые части (5) вычисляются для области $D\left(\epsilon_{n}, h\right)$.

Лемма 1. Справедливы равенства

$$
\begin{gathered}
t_{n}(+, \lambda, h)=t_{n}(-, \lambda, h), \\
\left|r_{n}( \pm, \lambda, h)\right|^{2}+\left|t_{n}( \pm, \lambda, h)\right|^{2}=1 .
\end{gathered}
$$

ДоКАЗАТЕЛЬСТВо. Проинтегрируем равенство

$$
u(+, \ldots) L_{x, y}\left(\epsilon_{n}, h\right) u(-, \ldots)-u(-, \ldots) L_{x, y}\left(\epsilon_{n}, h\right) u(+, \ldots)=0
$$

по области $x<R$, используем формулу Грина, граничные условия, асимптотику (5) и перейдем к пределу $R \rightarrow \infty$. В результате получим равенство (7). Равенство (8) есть следствие унитарности матрицы рассеяния и формул, связывающих матрицу рассеяния с коэффициентами отражения и прохождения. Лемма 1 доказана.

Положим по определению

$$
t_{n}(\lambda, h)=t_{n}( \pm, \lambda, h) .
$$


О СВЯЗИ МЕЖДДУ ПОЛЮСОМ МАТРИЦЫ РАССЕЯНИЯ И КОЭФФИЦИЕНТАМИ . . 307

\section{3. ПОВЕДЕНИЕ КОЭФФИЦИЕНТОВ ОТРАЖЕНИЯ И ПРОХОЖДЕНИЯ В ОКРЕСТНОСТИ РЕЗОНАНСА}

Наша задача состоит в изучении поведения коэффициентов $t_{n}(\lambda, h), r_{n}( \pm, \lambda, h)$ в окрестности точки $\lambda=\omega_{\text {res }}$ при $n \rightarrow \infty$. Покажем, что эта задача может быть рассмотрена в рамках предложенной в работе [3] модели. Для этого нужно сделать следующее.

1. В качестве пространства $H$ из работы [3] взять пространство $H=L^{2}\left(D\left(\epsilon_{1}, h\right)\right)$.

2. В качестве пространств $H_{ \pm}$из работы [3] взять пространства

$$
H_{ \pm}=L^{2}\left(D\left(\epsilon_{1}, h\right), e^{\mp|x|} d x d y\right)
$$

3. В качестве пространства $\Omega$ из работы [3] (пространства, параметризующего амплитуду рассеяния) нужно взять декартово произведение двухточечного множества \pm на множество целых чисел. Это ясно из формул (5).

4. В качестве оператора $A_{0}$ из работы [3] нужно взять оператор $A_{0}=-e^{-t L\left(0, h_{1}\right)}$, a в качестве операторов $A_{n}$ - операторы

$$
A_{n}=-e^{-t L\left(\epsilon_{n}, h\right)} .
$$

Выбор параметра $t>0$ пояснен в работе [4]. Годится любое $t>0$ за исключением, быть может, некоторого конечного множества.

5. В качестве отображения $U$ из работы [3] нужно взять отображение, которое диагонализует оператор $-e^{-t L\left(0, h_{1}\right)}$. Оно строится тривиально методом разделения переменных.

6. В качестве отображений $l, p$ из работы [3] нужно взять следующие пары:

$$
\begin{array}{ll}
l_{1}(\psi(x, y))=\overline{\psi(x, y)}, & p_{1}( \pm, n)=(\mp, n) \\
l_{2}(\psi(x, y))=\overline{\psi(-x, y)}, & p_{2}( \pm, n)=( \pm, n)
\end{array}
$$

В работе [4] показано, что при таком выборе выполнены все условия работы [3]. Заметим, что сделанные в работе [3] предположения о гладкости границы можно приспособить к рассматриваемому случаю с помошью хорошо известной техники винеровской аппроксимации, и мы не будем задерживать на этом внимание.

Пусть $\lambda_{n}$ - числа, определяемые формулами (9) и (24) из работы [3]. Положим

$$
\mu_{n}(h)=-\ln \left(-\frac{\lambda_{n}}{t}\right),
$$

если правая часть (12) вычисляется для области $D\left(\epsilon_{n}, h\right)$.

ЛЕмма 2. 1) Число $\mu_{n}$ есть полюс аналитического продолжения интегрального ядра резольвенты оператора $L\left(\epsilon_{n}, h\right)$.

2) Справедливы соотночения

$$
\operatorname{Im} \mu_{n}(h)<0, \quad \mu_{n}(h) \rightarrow \omega_{\text {res }}, \quad n \rightarrow \infty
$$


ДокАЗАТЕЛЬСТво. Первое утверждение следует из формулы (12) и определения числа $\lambda_{n}$ как полюса резольвенты оператора $A_{n}$. Второе утверждение вытекает из рассуждений, приведенных в работе [3] после формулы (24), и нашего предположения о том, что у операторов $L\left(\epsilon_{n}, h\right)$ нет собственных значений вблизи точки $\omega_{\text {res }}$. Лемма 2 доказана.

Из леммы 2 следует, что число $\mu_{n}(h)$ есть резонансный полюс для оператора $L\left(\epsilon_{n}, h\right)$ в принятом в теории дифференциальных уравнений смысле [5].

Положим по определению

$$
\begin{aligned}
\text { v.p. } t_{\infty}\left(\omega_{\text {res }}, h\right) & =\lim _{\left|\lambda-\omega_{\text {res }}\right| \rightarrow+0}\left(\lim _{n \rightarrow \infty} t_{n}(\lambda, h)\right), \\
\text { v. p. } r_{\infty}\left( \pm, \omega_{\text {res }}, h\right) & =\lim _{\left|\lambda-\omega_{\text {res }}\right| \rightarrow+0}\left(\lim _{n \rightarrow \infty} r_{n}( \pm, \lambda, h)\right) .
\end{aligned}
$$

Обратим внимание на то, что важен порядок предельных переходов в (14). Сушествование пределов доказано в работе [4].

Лемма 3. Справедливы соотношения

$$
\begin{aligned}
\text { v. p. } t_{\infty}\left(\omega_{\text {res }}, h_{1}\right)=1, & \text { v. p. } r_{\infty}\left( \pm, \omega_{\text {res }}, h_{1}\right)=0, \\
\text { v. p. } t_{\infty}\left(\omega_{\text {res }}, 0\right)=0, & \mid \text { v. p. } r_{\infty}\left( \pm, \omega_{\text {res }}, 0\right) \mid=1 .
\end{aligned}
$$

ДокАЗАТЕЛЬСТво. Если резонатор закрыт (т.е. $n=\infty$ ), то очевидно, что при $\lambda \neq$ $\omega_{\text {res }}$ справедливы равенства

$$
t_{\infty}\left(\lambda, h_{1}\right)=1, \quad t_{\infty}(\lambda, 0)=0
$$

Отсюда и из равенства (8) следует утверждение леммы 3.

Теорема. Пусть последовательность действительных чисел $\gamma_{n}(h)$ удовлетворяет условию

$$
\left|\omega_{\text {res }}-\gamma_{n}(h)\right|=O\left(\left|\operatorname{Im} \mu_{n}(h)\right|^{1+\delta}\right), \quad \delta>0, \quad n \rightarrow \infty .
$$

Тогда

$$
\left|t_{n}\left(\gamma_{n}(0), 0\right)\right| \rightarrow 1, \quad\left|t_{n}\left(\gamma_{n}\left(h_{1}\right), h_{1}\right)\right| \rightarrow 0, \quad n \rightarrow \infty .
$$

ДокАЗАТЕЛЬСТво. В случае $h=h_{1}$ применим теорему 2 из работы $[3]$ при $l=l_{1}$, $p=p_{1}$. Используя равенство (15), получим

$$
\lim _{n \rightarrow \infty}\left(\left|r_{n}\left(+, \gamma_{n}\left(h_{1}\right), h_{1}\right)\right|+\left|r_{n}\left(-, \gamma_{n}\left(h_{1}\right), h_{1}\right)\right|\right)=2 .
$$

Так как

$$
\left|r_{n}\left( \pm, \gamma_{n}\left(h_{1}\right), h_{1}\right)\right| \leqslant 1
$$


то из (17) следует, что

$$
\lim _{n \rightarrow \infty}\left|r_{n}\left( \pm, \gamma_{n}\left(h_{1}\right), h_{1}\right)\right|=1
$$

и поэтому

$$
\lim _{n \rightarrow \infty}\left|t_{n}\left(\gamma_{n}\left(h_{1}\right), h_{1}\right)\right|=1
$$

Во втором случае $(h=0)$ мы воспользуемся симметрией задачи относительно отражений $(x, y) \rightarrow(-x, y)$ и в теореме 2 из работы [3] положим $l=l_{2}, p=p_{2}$. Далее будем рассуждать аналогично первому случаю. Теорема доказана.

Отметим, что задача о взаимодействии резонатора и волновода в случае иной геометрии рассмотрена в работе [6]. В работе [7] для другой ситуации и другим методом доказано, что коэффициент прохождения обрашается в нуль на частоте резонатора.

Благодарности. Работа выполнена при поддержке РФФИ, проект № 02-01-00271.

\section{Список литературы}

[1] P. Mavropoulos, N. Papanikolaou, P. H. Dederichs. A KKR Green formalism for ballistic transport. cond-mat/0306604.

[2] F.-M. Dittes. Phys. Rep. 2000. V. 339. P. 215-316.

[3] А. А. Арсеньев. ТМФ. 2003. Т. 134. № 3. С. 341-352.

[4] А. А. Арсеньев. Матем. сб. 2003. Т. 194. № 1. С. 3-22.

[5] J. Sjöstrand, M. Zworski. J. Am. Math. Soc. 1991. V. 4. P. 729-769.

[6] H.-J. Stöckmann, E. Persson, Y.-H. Kim, M. Barth, U. Kuhl, I. Rotter. Phys. Rev. E. 2002. V. 65. P. 066211-1-066211-9.

[7] W. Porod, Z. Shao, C. S. Lent. Appl. Phys. Lett. 1992. V. 61. № 11. P. 1350-1352.

Поступила в редакцию $3 . \mathrm{X.2003} \mathrm{г.}$ 